\title{
A fully 3-dimensional thermal model of a comet nucleus
}

\author{
Eric D Rosenberg and Dina Prialnik \\ Department of Geophysics and Planetary Sciences, \\ Raymond and Beverly Sackler Faculty of Exact Sciences, \\ Tel-Aviv University, \\ Tel-Aviv 69978, Israel.
}

\begin{abstract}
A 3-D numerical model of comet nuclei is presented. An implicit numerical scheme was developed for the thermal evolution of a spherical nucleus composed of a mixture of ice and dust. The model was tested against analytical solutions, simplified numerical solutions, and 1-D thermal evolution codes. The 3-D code was applied to comet $67 \mathrm{P} /$ Churyumov-Gerasimenko; surface temperature maps and the internal thermal structure was obtained as function of depth, longitude and hour angle. The effect of the spin axis tilt on the surface temperature distribution was studied in detail. It was found that for small tilt angles, relatively low temperatures may prevail on near-pole areas, despite lateral heat conduction. A high-resolution run for a comet model of $67 \mathrm{P} /$ Churyumov-Gerasimenko with low tilt angle, allowing for crystallization of amorphous ice, showed that the amorphous/crystalline ice boundary varies significantly with depth as a function of cometary latitude.
\end{abstract}

Key words: 3-D numerical model, comet nucleus, thermal evolution PACS: $96.30 . \mathrm{Cw}$

\section{Introduction}

Our knowledge of the nature of comets, their structure, composition, dynamical history, and modes of activity has greatly improved over the last twenty

Email addresses: erosenbe@post.tau.ac.il (Eric D Rosenberg), dina@planet.tau.ac.il (Dina Prialnik).

URL: http://geophysics.tau.ac.il/personal/erosenbe (Eric D Rosenberg). 
years. The one event that constituted a mile-stone in the study of comets was the space mission Giotto, which provided the first close-up picture of a comet's heart - its nucleus. Several other space missions to comets followed, crowned by the very recent Deep Impact mission (A'Hearn et al., 2005), aimed at revealing properties of the nucleus interior. As observational data on comets has been accumulating, theoretical studies and modeling have started to develop, in order to interpret, predict and explain new findings and discoveries.

Comets consist of frozen gases and dust. The dust consists of silicates and organic materials, while the frozen gases are mostly water and a few constituents more volatile than water. Solar heat, not reflected or radiated from the surface of the nucleus is primarily used to evaporate ices from exposed areas. The remainder of the heat penetrates into the nucleus, where it can cause numerous physico-chemical reaction processes, which eventually affect measurable properties at the nucleus surface. Over the past two decades, numerical studies have attempted to integrate some of the more influential processes with a numerical representation of a comet nucleus, in order to better understand the rather unpredictable behavior of comets (Prialnik et al., 2004).

The most obvious property of comet nuclei is the lack of spherical symmetry. Besides the fact that self-gravity, which dictates the spherical shape of larger celestial bodies, is negligible in comets, the main energy source - solar radiation - is unevenly distributed over the nucleus surface, not only because of daily variation and spin axis inclination, but also because of the large orbital variations, characteristic to comets, but not to other bodies of the solar system. Nevertheless, theoretical models of nuclei have so far assumed spherical symmetry, or have circumvented this assumption by crude approximations.

Modeling began with very simple pictures of the nucleus. A few physical processes with simplifying assumptions provided crude but insightful explanations for the comet nucleus behavior (Cowan and Ahearn, 1979; Fanale and Salvail, 1984; Herman and Podolak, 1985; Prialnik and Bar-Nun, 1987). However, these models were able to give only average results and explain only the general behavior of comets. The models were limited to a single dimension, mostly based on spherical symmetry (the fast rotator approximation). They simulated heat flow in a mixture of ice and rock (dust) with uniform boundary conditions for the solar flux, radiative emission and substance sublimation. Additional physico-chemical processes, e.g., amorphous ice crystallization, surface erosion etc., were added later on to produce a more elaborate physical picture. As these models did not take into consideration spatial variations in surface temperature, they provided a fair approximation only for the internal part of the nucleus, where these variations fade.

For more realistic surface results, slow rotator approaches were adopted. A "1.5-D" model (Benkhoff and Boice, 1996; Benkhoff, 1999) was suggested, in 
which, a 1-D model was applied with boundary conditions of an equatorial point on a rotating nucleus. This way, diurnal boundary conditions differences were taken into considerations, and an upper limit for production rate was achieved when the sub-solar (high noon) flux was adopted for the entire surface of the sunlit hemisphere. This model, as its predecessor, does not calculate lateral flow. A semi-lateral approach was taken for the 2.5-D model (Enzian et al., 1997, 1999) where a meridian flow was calculated, and boundary conditions were taken as in the 1.5-D model.

A different approach is the quasi-3-D procedure (Gutiérrez et al., 2000; Julian et al., 2000; Cohen et al., 2003). In this approach, every point on the nucleus surface is calculated (with the appropriate local boundary condition) with radial flow only, so calculations are similar to the 1.5-D method, but boundary conditions are of the total sphere, just as with a 3-D approach. Still, each point (or element) of the surface evolves independently of the others and only radial conduction is considered.

Now that modeling has made significant progress towards understanding the general structure and behavior of comets, more sophisticated and realistic pictures of the nucleus are required.

The purpose of the present research project is to develop a fully 3-D model of a comet nucleus. As in the quasi-3-D, boundary conditions will be taken for the entire sphere, but now, meridional and azimuthal heat fluxes are calculated as well. Gas production and flow in the interior of the comet are not included in the present model. In order to compare production rates of volatiles with the observed ones at large distances from the nucleus, when the surface is not resolved (in fact, not even seen) (Biver et al., 1999), a sum of all local production rates can be calculated. However, the model will provide a detailed description of the surface activity as well.

In the 3-D model, in contrast to the older models, all the grid elements need to be solved simultaneously, so the computational load increases dramatically. This may limit the spatial resolution of the comet if we require a solution in a reasonable time. However, as modern computers advance rapidly, the grid density may increase for the same calculation time. This model may be scaled for super-computers to allow even denser grid resolution.

\section{Model description}

Our purpose is to calculate numerically a variety of physical processes that are believed to take place inside a comet nucleus. In order to do so, physical equations and models should be adjusted for discrete calculations. The basic 
adjustment is dividing the nucleus into elements via a grid, and simplifying the problem by assuming a "homogeneous lumped system" approximation for each element.

\subsection{3-D heat conduction equation}

Heat conduction is modeled by Fourier's law

$$
\mathbf{F}=-K \nabla T
$$

where $\mathbf{F}$ is the heat flux, $K$ is the conduction coefficient and $T$ is the temperature.

In 3-D spherical coordinates, equation (1) will take the form

$$
\mathbf{F}=-H_{z} K\left(\frac{\partial T}{\partial r} \hat{\boldsymbol{r}}+\frac{1}{r} \frac{\partial T}{\partial \theta} \hat{\boldsymbol{\theta}}+\frac{1}{r \sin \theta} \frac{\partial T}{\partial \phi} \hat{\boldsymbol{\phi}}\right)
$$

where $H_{z}$ is the Hertz factor, a correction factor $(<1)$ for the heat conductivity of porous substances that accounts for the reduced contact area between solids as compared to the cross-sectional area. In order to calculate the effective conduction coefficient $K$ for a "homogeneous lumped system" we considered all substances distributed evenly through the system, and the contribution of each substance to the final value is taken to be proportional to its mass fraction $X_{\alpha}$. Thus

$$
K=\sum_{\alpha} X_{\alpha} K_{\alpha}
$$

where $K_{\alpha}$ is the conduction coefficient of substance $\alpha$.

When all fluxes through a "lumped system" are combined we get the heat equation

$$
-\boldsymbol{\nabla} \cdot \mathbf{F}+q=\rho C_{p} \frac{\partial T}{\partial t},
$$

where $q$ is the rate of energy release by an internal heat source within the "lumped system", $\rho$ is the bulk density and $C_{p}$ is the heat capacity.

\subsubsection{Amorphous to crystalline ice transition}

The crystallization process of amorphous ice is spontaneous but greatly affected by temperature. Its rate is given by (Schmitt et al., 1989), based on laboratory experiments

$$
\lambda=1.05 \times 10^{13} \mathrm{e}^{-5370 / T} \quad\left[s^{-1}\right]
$$


The amorphous ice mass fraction $X_{A}$ changes with time at a rate

$$
\dot{X}_{A}=-\lambda(T) X_{A}
$$

This process, which changes the ice structure, also changes its physical properties, such as density and thermal conductivity. In addition, crystallization is an exothermic process that can cause chain-reaction, that is, propagate by feeding on its own energy. The rate of heat generated by crystallization is given by

$$
q=\lambda(T) \rho X_{A} \mathcal{H}_{a}
$$

where $\mathcal{H}_{a}$ is the energy released per unit mass (Ghormley, 1968).

\subsection{Boundary conditions}

Boundary conditions at the surface consist of balance of heat fluxes into the nucleus from external sources and out of it into space. Three such sources are considered:

(1) Solar radiation flux, given by

$$
(1-\mathcal{A}) \frac{L_{\odot}}{4 \pi d_{H}^{2}} \cos \xi
$$

where $\mathcal{A}$ is the surface albedo, $L_{\odot}$ is the solar luminosity, $d_{H}$ is the heliocentric distance and $\xi$ is the local solar zenith angle. Here $\cos \xi$ is calculated for surface coordinates $\theta$ and $\phi$ according to

$$
\cos \xi=\sin \theta_{s} \sin \theta \cos \left(\phi_{s}+\phi\right)+\cos \theta_{s} \cos \theta,
$$

where $\theta_{s}$ and $\phi_{s}$ are the sub-solar coordinates.

(2) Thermal radiation loss, given by

$$
\epsilon \sigma T^{4}
$$

where $\epsilon$ is the emissivity and $\sigma$ is the Stefan-Boltzmann constant.

(3) Latent heat absorbed per unit time by sublimation of volatiles, given by

$$
\mathcal{P}_{v}(T) \sqrt{\frac{m}{2 \pi k T}} H,
$$

where $m$ is the molecular weight, $H$ is the latent heat of sublimation and $\mathcal{P}_{v}$ is the vapor pressure, given by the Clausius-Clapeyron equation

$$
\mathcal{P}_{v}=a \mathrm{e}^{-b / T}
$$



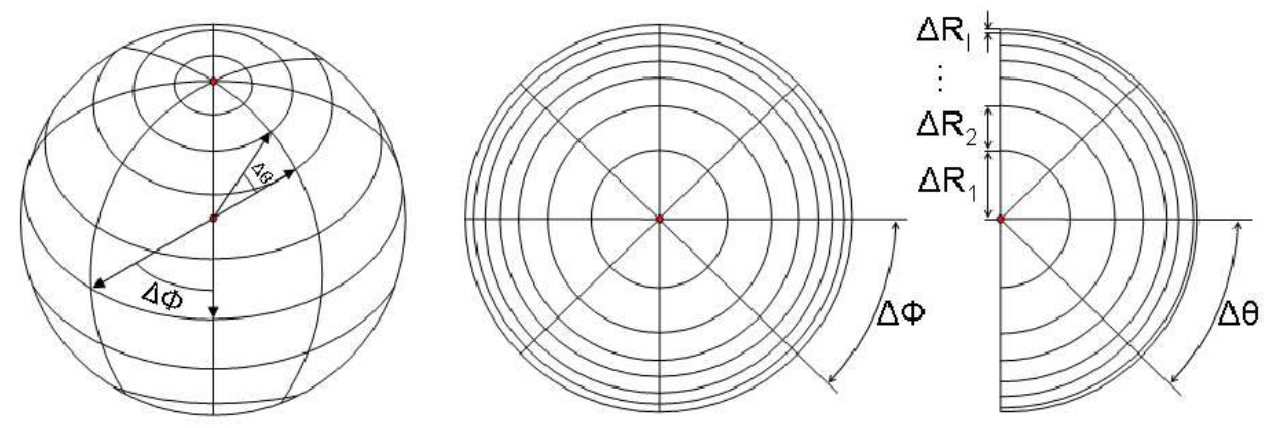

Fig. 1. Logical representation of the numerical grid, conforming to spherical coordinates convention. The $\Theta$ coordinate is divided into ' $J$ ' equal intervals $(\Delta \Theta)$, the $\Phi$ coordinate is divided into ' $K$ ' equal intervals $(\Delta \Phi)$ and the radial coordinate is divided with geometric progression into ' $I$ ' intervals $\left(\Delta R_{i}\right)$. The equations of the numerical scheme are based on this representation.

\subsection{Numerical scheme}

The heat equation (4) is a non-linear second-order partial differential equation, which has no analytical solution and has to be solved numerically. The differential equation is transformed into a set of difference equations, and applied to a finite grid, where the infinitesimals $\delta R, \delta \Theta, \delta \Phi$ and $\delta t$ become the grid steps $\Delta R, \Delta \Theta, \Delta \Phi$ and $\Delta t$, respectively.

The grid has two representations, the logical (geometric) one and the computerized (algebraic) one. The logical grid, shown in Fig. 1, is the spatial representation of the difference equations, and is a 3-D grid in spherical coordinates. The grid itself is a 3-D mesh with $I, J$ and $K$ divisions for $R$ (radial distance), $\Theta$ (co-latitude) and $\Phi$ (azimuth). In order to better study surface phenomena, $\Delta R$ is taken to be decremental geometrically, and is uniquely determined by the radius of the nucleus, the number of divisions (' $I$ ') and the surface layer's thickness. Dimensions $\Theta$ and $\Phi$ are divided into equal intervals.

The computerized grid is a vector with $n \equiv I \cdot J \cdot K$ elements. The elements are organized along the vector, as shown in Fig. 2, so that radius values will vary the slowest, and the co-latitude will vary the fastest. The relation between the logical and the computerized grid is expressed by:

$$
v=j+(k-1) J+(i-1) J K
$$

$v$ is the position in the computer grid vector, while $i, j$ and $k$ are the indexes for $R, \Theta$ and $\Phi$ and $J$ and $K$ are number of divisions for $\Theta$ and $\Phi$. This configuration forms a diagonal equation matrix, which is easier to solve. 


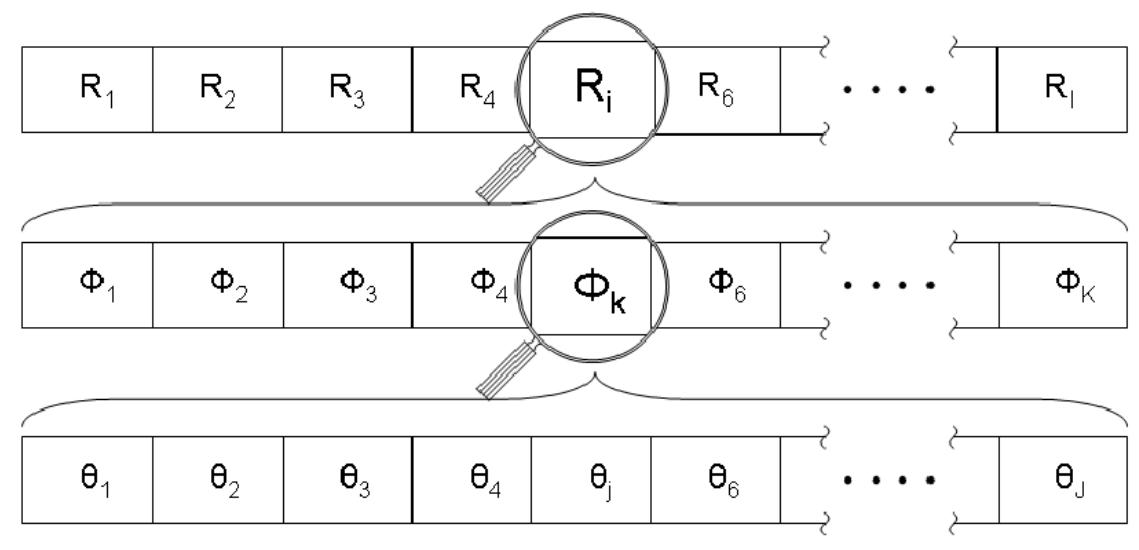

Fig. 2. Computerized (vector) representation of the numerical grid. This representation is the order of the grid's elements in the solution vector of size $n \equiv I \cdot J \cdot K$. The relation between the logical and vector grids is given by equation (13). In this representation $R$ varies the slowest through the vector and $\Theta$ varies the fastest. The equations matrix, according to this representation, will be diagonal (albeit not tight) and easier so solve.

\subsection{Difference equations}

For greater stability, and in order to remove time step constraints, a fully implicit iterative scheme is chosen, although the formulation is more complicated and has a higher computational load. The difference equation for each volume element has the form

$$
\sum F_{x}^{(n)} \Delta S_{x}+q^{(n)} \Delta V=\frac{U^{(n)}-U^{(0)}}{\Delta t} \Delta V
$$

where $x$ is the direction $\left(i^{+}, i^{-}, j^{+}, j^{-}, k^{+}\right.$and $\left.k^{-}\right), n$ is the iteration number, $F_{x}$ is the heat flux vector component in the $x$ direction, $\Delta S_{x}$ is the element's $x$ side area, $q^{n}$ is produced heat inside the element, $\Delta V$ is the element's volume, $\Delta t$ is the time step, and $U$ is the thermal energy. We note that the difference scheme is conservative, that is, upon integration over the entire volume, all fluxes cancel out except the flux crossing the nucleus surface. In other words, Gauss's theorem is satisfied by the difference equations on the discrete grid. The sum on the LHS of equation (14) runs over all sides of a volume element: 6 for most elements, 5 for those ending on the $\Theta=0$ or $\pi$ axis, and 4, for those ending at the center $R=0$.

The equations are linearized and solved for $\Delta T$ rather than for $T$ itself; as $\Delta T$ reduces over iterations, it serves as convergence criterion. 


\section{Tests of the $3-\mathrm{D}$ model}

\subsection{Analytical and numerical tests}

Several comparative tests were carried out to ensure that heat flow is correctly solved. First, two analytical solutions for the heat transport equation on a sphere were chosen. Since all terms in our surface boundary condition (solar radiation, sublimation and thermal emission) are non-linear, and hence do not allow analytical solutions to the heat equation, we chose a linear convective boundary condition. The numerical model was modified to the same boundary condition, and kept spherically symmetric in order to become comparable to the analytical solution. The first analytical model (hereafter, C\&J) was taken from the literature (Carslaw and Jaeger, 1959):

$$
T(r, t)=\frac{2 h T_{0}}{r} \sum_{n} \frac{\sin \left(\lambda_{n} a\right)\left[\left(\lambda_{n} a\right)^{2}+(1-a h)^{2}\right]}{\lambda_{n}^{2}\left[\lambda_{n}^{2}-a h(1-a h)\right]} \sin \left(\lambda_{n} r\right) e^{-\lambda_{n}^{2} D t}
$$

$\lambda_{n}$ defined as:

$$
\tan \left(\lambda_{n} a\right)=\frac{\lambda_{n} a}{1-a h}
$$

where $T_{0}$ is the initial temperature, $a$ is the sphere's radius, $h$ is the convection constant, $D$ is the conduction constant and $t$ is the time step; $\lambda_{n}$ is the series solution of Eq.(16). This solution is based on simplifying assumptions.

A second, accurate analytical solution was derived:

$$
T(r, t)=\frac{2 T_{0}}{r} \sum_{n} \frac{\sin \left(\lambda_{n} a\right)-\left(\lambda_{n} a\right) \cos \left(\lambda_{n} a\right)}{\lambda_{n}^{2} a-\lambda_{n} \sin \left(\lambda_{n} a\right) \cos \left(\lambda_{n} a\right)} \sin \left(\lambda_{n} r\right) e^{-\lambda_{n}^{2} D t}
$$

which is independent of $h$, and therefore not limited by boundary condition values.

A different test model was obtained by solving the heat equation numerically by a $1-D$ spherical explicit scheme:

$$
T_{i}^{(n+1)}=T_{i}^{(n)}+2 D \frac{\Delta t}{\Delta V_{i}}\left[\frac{T_{i-1}^{(n)}-T_{i}^{(n)}}{\Delta r_{i}+\Delta r_{i-1}} S_{i}+\frac{T_{i+1}^{(n)}-T_{i}^{(n)}}{\Delta r_{i}+\Delta r_{i+1}} S_{i+1}\right]
$$

with boundary condition $(i=I)$ :

$$
T_{I}^{(n+1)}=T_{I}^{(n)}+2 D \frac{\Delta t}{\Delta V_{I}}\left[\frac{T_{I-1}^{(n)}-T_{I}^{(n)}}{\Delta r_{I}+\Delta r_{I-1}} S_{I}-\frac{1}{2} h T_{s}^{(n)} S_{I+1}\right]
$$



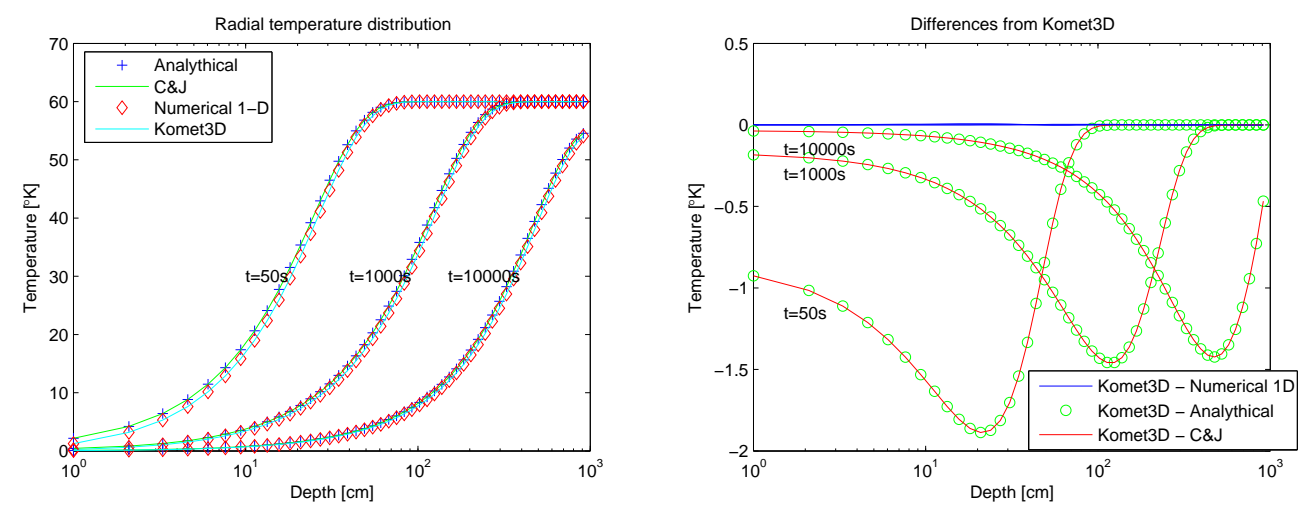

Fig. 3. Different solutions for a cooling sphere with uniform initial conditions, and linear convection boundary condition, at 50, $1000 \& 10000$ seconds. All solutions: Carslaw \& Jaegar, analytical development, numerical 1-D and the 3-D (Komet3D) models converge to the same result (left). The absolute difference between all models to the 3-D model (right) reveals perfect agreement between the numerical models, and up to $5 \%$ error for the analytical solutions.

where

and

$$
\Delta V_{i}=\frac{4}{3} \pi\left[\left(R_{i}+d R_{i}\right)^{3}-R_{i}^{3}\right]
$$

$$
S_{i}=4 \pi R_{i}^{2}
$$

The tests were performed on a sphere with $R=1000 \mathrm{~cm}$. All other parameters were chosen artificially (to allow the C\&J model to converge) and do not represent a comet nucleus. The sphere was initially set at a uniform temperature of $60^{\circ} \mathrm{K}$ and was then allowed to cool. Temperature profiles as function of depth are shown in Fig. 3 at three different times for all 4 solutions. Clearly, the 3-D model agrees very well with both analytical models, as well as with the 1-D numerical model at all times. A more detailed comparison of the model with the other models is obtained by plotting the differences between them, as shown in the right panel of Fig. 3. The maximal difference between the $3-\mathrm{D}$ model and the analytical models is less than $5 \%$, which is an excellent agreement, keeping in mind that the lowest radial grid resolution is $9 \%$; the difference between the two numerical models is negligibly small.

\subsection{Comparison with other models for comet 46P/Wirtanen}

Comet 46P/Wirtanen was intensively studied while it was the target of space mission Rosetta. In particular, several different and independent numerical codes were applied to this comet and the results were carefully analyzed and compared (Huebner et al. 1999). These models, allowing for differences among them, can be considered a reliable test for the present model. We therefore used our code adopting comet 46P/Wirtanen's parameters ( $a=3.093738$ AU, 

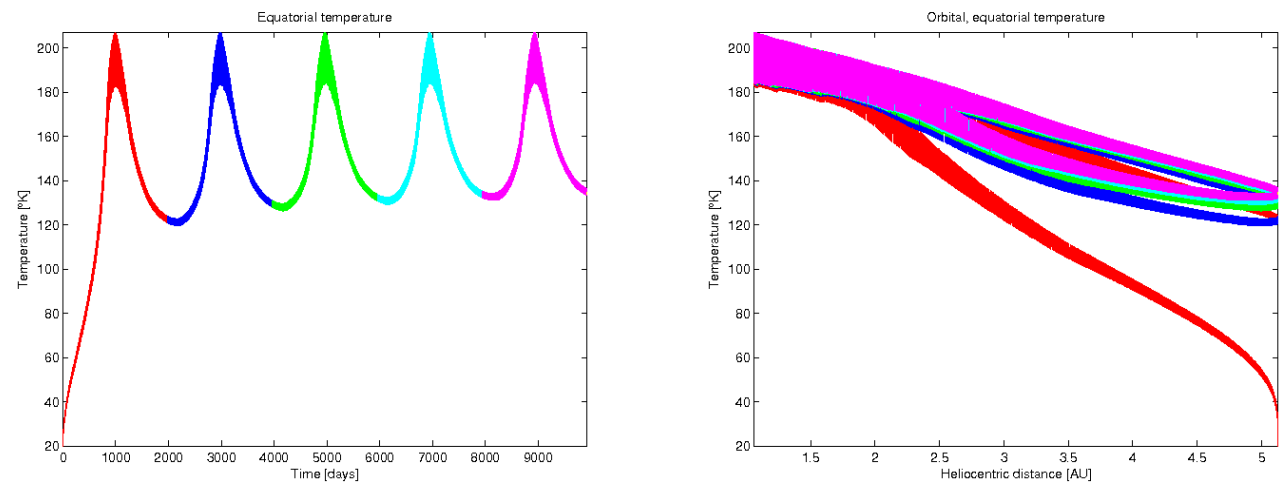

Fig. 4. Orbital temperature variation of an equatorial model element with comet Wirtanen's orbital parameters, for 5 revolutions around the sun. Colors distinguish between revolutions. Line thickness indicates temperature differences between day and night sides.

$\mathrm{e}=0.6578222, \mathrm{R}=600 \mathrm{~m}$, and a spin period of $24 \mathrm{hr}$, as used in other model calculations), and calculated the thermal evolution for 5 revolution around the sun, assuming the spin axis to be perpendicular to the orbital plane (zero tilt angle). The nucleus composition consisted of $50 \%$ crystalline $\mathrm{H}_{2} \mathrm{O}$ ice and $50 \%$ dust, by mass. No Hertz factor was used $\left(H_{z}=1\right)$. In Fig. 4 , the temperature of a point on the equator is shown versus time and heliocentric distance. The line thickness represents daily temperature variations. As expected, the perihelion (daytime) temperature is the same for all orbits, since heat exchange with the interior is negligible in this case, and the surface temperature is controlled by sublimation. The aphelion temperature increases slightly with each period; again, this is to be expected, as the nucleus surface layer accumulates heat and needs several revolutions to stabilize. The results are in very good agreement with Huebner et al. (loc. cit.), for a model with similar parameters. We also compared our results with the quasi-3-D model of comet 46P/Wirtanen computed by (Cohen et al., 2003) and found very good matching of the temperature map.

Fig. 5 illustrates boundary fluxes for that same point on the equator. Positive fluxes are directed inward, and negative fluxes outward. At perihelion, the solar flux is mostly absorbed by sublimation, while at aphelion the various terms are comparable. The residual flux, which represent heat conducted into or out of the nucleus, shows that on the day side, there is a net inward flux, and on the night side, a net outward flux. 


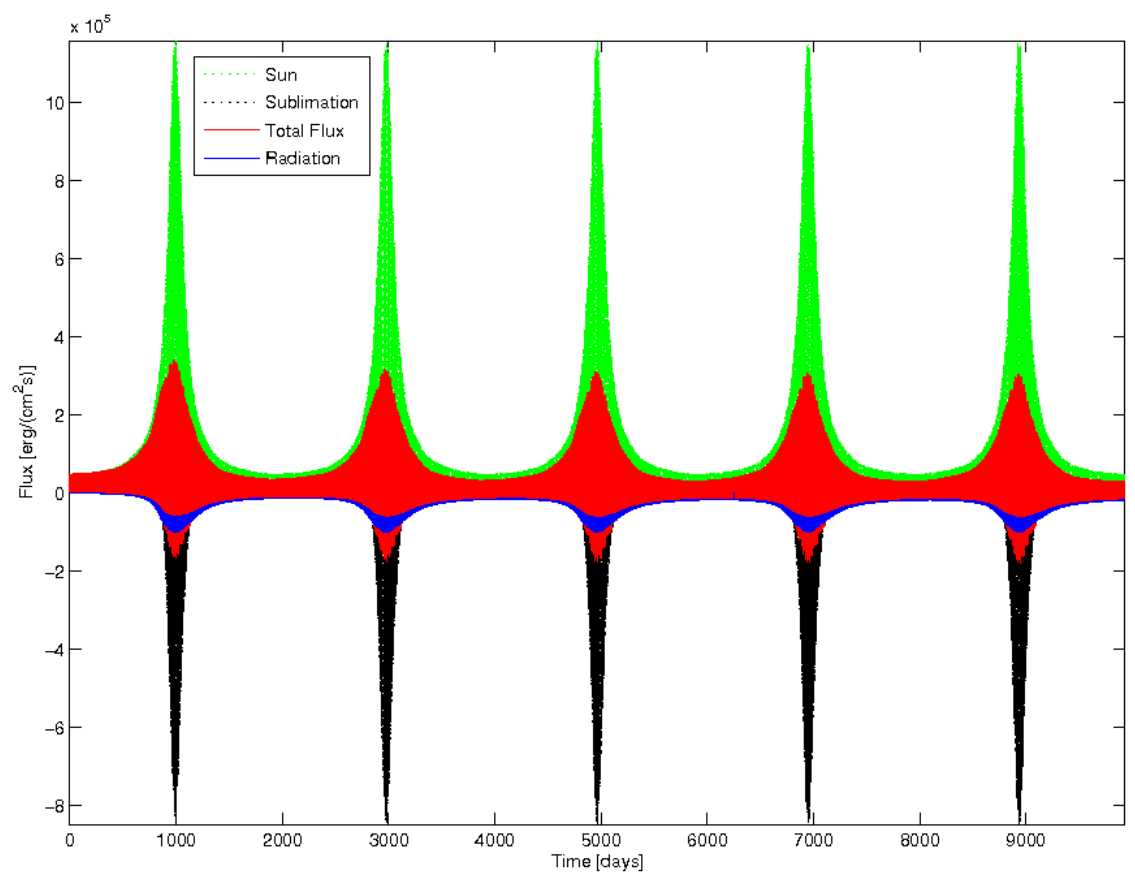

Fig. 5. Surface flux balance for equatorial element, with comet Wirtanen's orbital parameters (same as in Fig. 4). Positive values are inward fluxes, and negative are outwards fluxes (relative to the nucleus center). The red line represents total flux.

\section{Applications of the 3-D code}

\subsection{Models of Comet 67P/Churyumov-Gerasimenko}

The actual target of the Rosetta mission is now comet 67P/ ChuryumovGerasimenko (hereafter, 67P/C-G). We have used our code to model the evolution of a spherical nucleus that has the characteristics of this comet, $\mathrm{a}=3.5029497 \mathrm{AU}, \mathrm{e}=0.6319359, \mathrm{R}=1980 \mathrm{~m}$, and a spin period of $12.6 \mathrm{hr}$ (Lamy et al., 2004), in particular a spin axis tilt of $40^{\circ}-45^{\circ}$ (Chesley, 2004). We considered a nucleus containing 50\% water crystalline ice and $50 \%$ dust by mass, and calculated the thermal evolution for 5 orbital revolutions. Figures 6 and 7 illustrate the daily and orbital skin depths, by comparing several temperature maps for different depths and orbital positions. Analytical diurnal and orbital skin-depths were calculated to be $11 \mathrm{~cm}$ and $8 \mathrm{~m}$ respectively, in good agreement with the numerical results.

Another model was calculated assuming an initial composition of 50\% amorphous ice and $50 \%$ dust by mass and allowing for crystallization of the amorphous ice. The water production rate over the entire nucleus surface is shown in Fig. 8 together with the respective temperature map at two heliocentric 

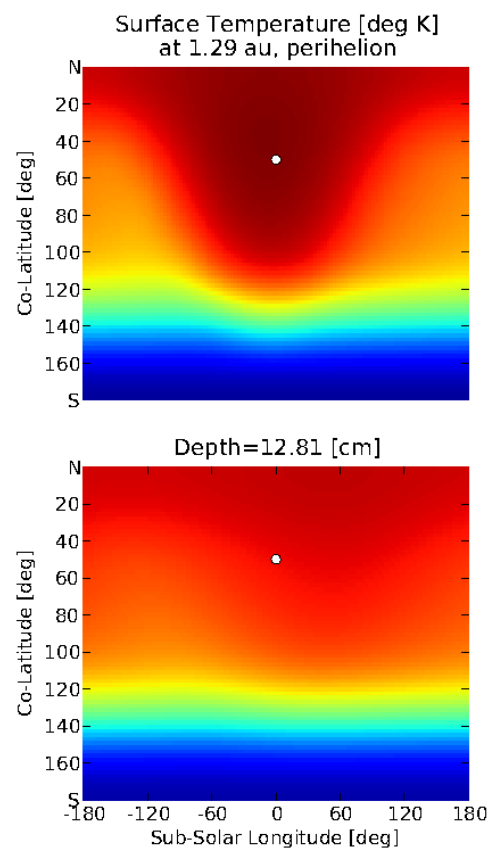
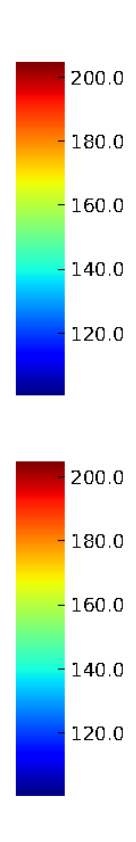

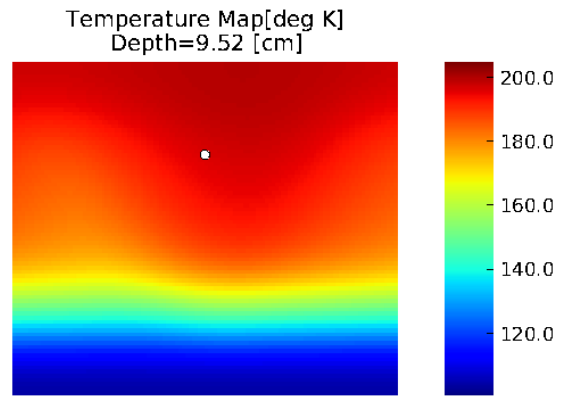

Depth $=16.89[\mathrm{~cm}]$

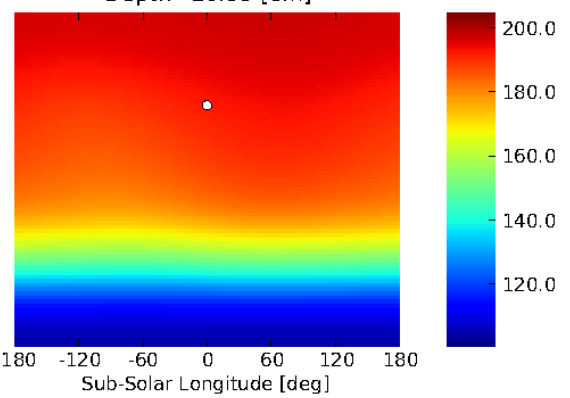

Fig. 6. Shell temperatures for different depths for comet $67 \mathrm{P} / \mathrm{C}-\mathrm{G}$ with $40^{\circ}$ tilt angle. The theoretical daily skin-depth for 50\% crystalline water and $50 \%$ dust with Hertz factor of $H_{z}=0.1$, was calculated to be $11 \mathrm{~cm}$. The maps indicates that the azimuthal temperature homogeneity increases with depth, and that deeper than $16 \mathrm{~cm}$, hourly temperatures are almost indistinguishable.

distances post-perihelion. The strong temperature dependence of the sublimation rate is illustrated by the high concentration of the active spot. We note that the peak is slightly shifted beyond the subsolar point (toward afternoon). The maximal integrated total production rate obtained (near perihelion), $\left.8.3 \cdot 10^{28} \mathrm{~s}^{-1}\right]$, is higher than the observed value of $\sim 1 \cdot 10^{28} \mathrm{~s}^{-1}$ (Schleicher and Millis, 2003); this is not surprising since the model assumes water sublimation from the entire surface, while active areas are most probably confined to a fraction of the nucleus surface, as indicated by close-up observations of comet nucleus surfaces (Keller et al., 1986; Sunshine et al., 2006).

\subsection{The effect of the spin-axis tilt}

Variations of an input parameter may lead to different behavior patterns for models that otherwise have the same properties. Here we show, for the first time, the effect of the spin-axis tilt on the temperature distribution over the surface of a short-period comet. For illustration, we have chosen the orbital parameters of comet $67 \mathrm{P} / \mathrm{C}-\mathrm{G}$. Our aim is to determine the minimal and maximal temperatures that can be obtained locally, under all possible inclina- 

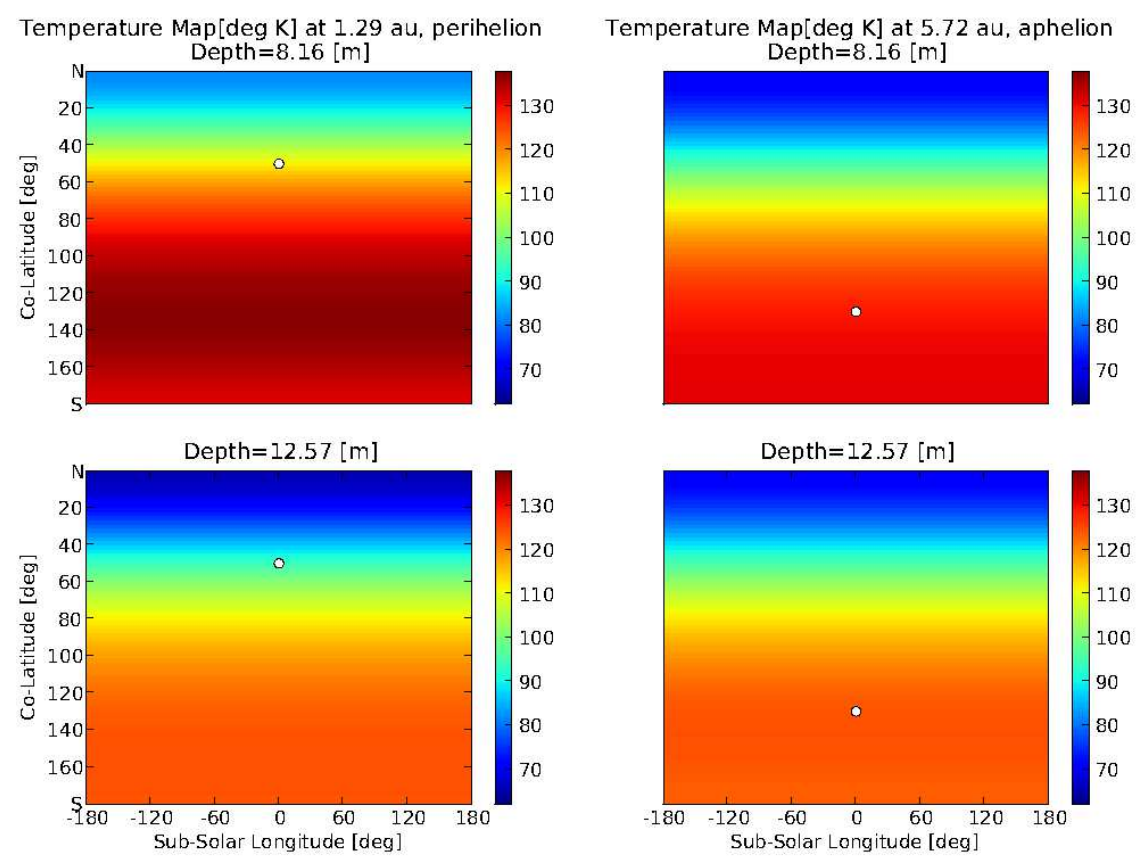

Fig. 7. Orbital skin-depth for comet $67 \mathrm{P} / \mathrm{C}-\mathrm{G}$ with $40^{\circ}$ tilt angle. For reference, the analytical value for $50 \%$ crystalline water and $50 \%$ dust with $H_{z}=0.1$ is $8 \mathrm{~m}$. Top maps show a spherical shell, $8 \mathrm{~m}$ deep, at perihelion (left) and at aphelion (right). Differences between them indicate that this depth is within the orbital skin-depth. The bottom maps show temperatures of a deeper shell $(12.5 \mathrm{~m})$, where differences are minute, indicating that the shell is deeper than the orbital skin-depth.

tions. This will shed light on the viability of very volatile ices on the surface of comet nuclei.

Assuming a spherical comet nucleus with the orbital parameters of comet $67 \mathrm{P} / \mathrm{C}-\mathrm{G}$, we carried out evolutionary calculations over the entire range of tilt angles, at intervals of $10^{\circ}$. For all models, the summer solstice point was taken to be at perihelion, (i.e. the projection of the spin vector on the ecliptic plane, points to the sun at perihelion). Thermal evolution was calculated over a period of 5 orbital revolution. The nucleus composition was taken to be $50 \%$ dust and $50 \%$ crystalline $\mathrm{H}_{2} \mathrm{O}$ ice by mass. Since we were interested in the temperature distribution at the surface, and since for a short-period comet, amorphous ice is present only at relatively large depths, we have assumed crystalline ice throughout. We adopted a 0.1 Hertz factor.

The results are summarized in Fig. 9. For maximum temperatures (left), southpole temperatures were always lower than the north-pole ones; however, for small tilt angles (below $40^{\circ}$ ) the difference was found to be more significant. When the tilted nucleus is at perihelion, the south-pole is directed away from the sun, not receiving any solar flux. The only heat flowing into the south pole is from non-lateral conduction, which is negligible relative to the solar 

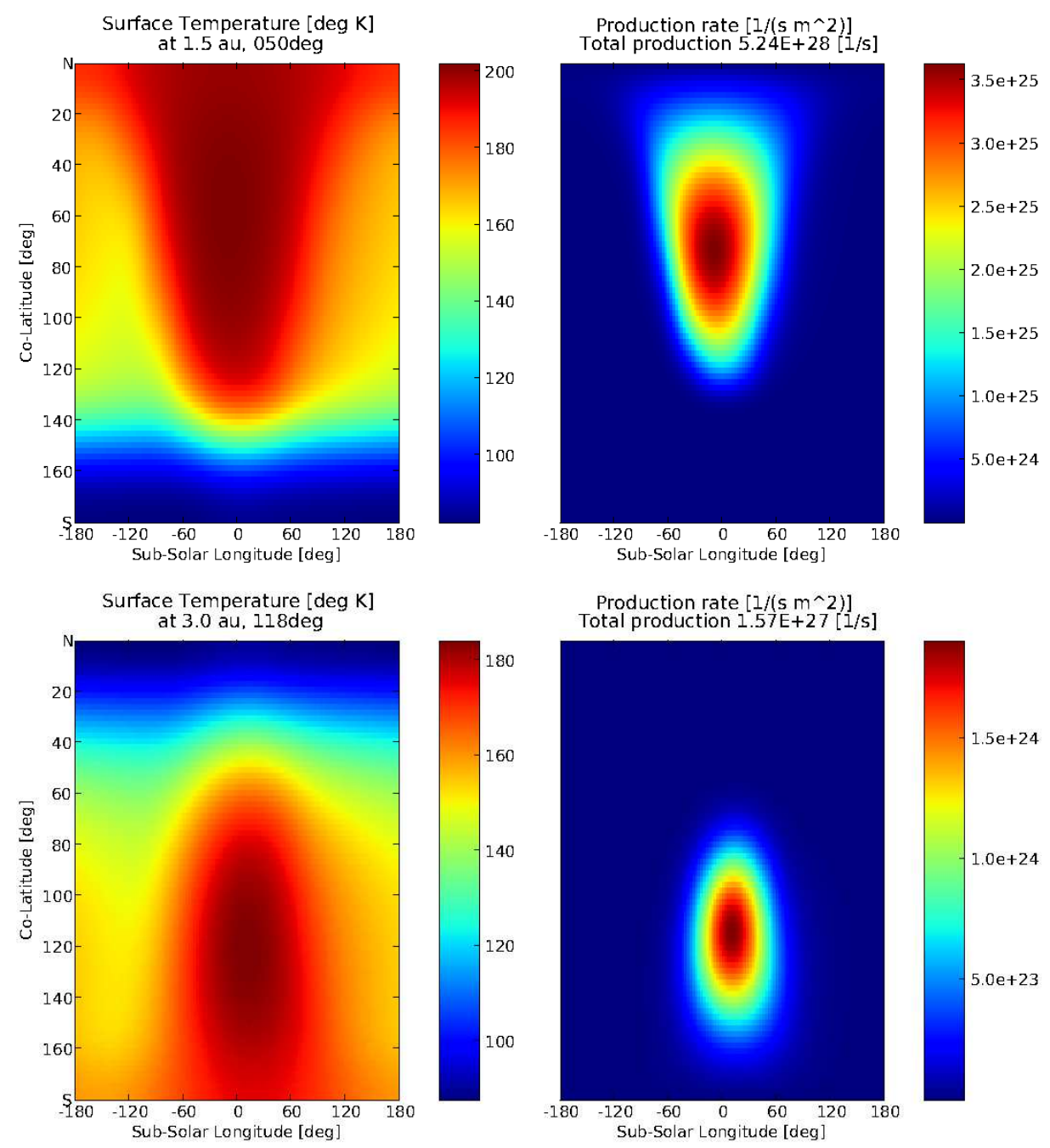

Fig. 8. Surface temperature and water production rate maps for comet $67 \mathrm{P} / \mathrm{C}-\mathrm{G}$ with $45^{\circ}$ tilt angle, where crystallization is taken into account. Top maps show temperature (left) and local flux $\left(\mathrm{m}^{-2} \mathrm{~s}^{-1}\right)$ of water molecules (right) at $1.5 \mathrm{AU}$ post-perihelion, and bottom maps, at 3 AU post-perihelion.

flux. The south-pole remains hidden from solar flux until the nucleus nears the equinox, where the sun is directly above the nucleus' equator, and the south pole is exposed to the sun at a very low angle. Passing the equinox point, the south pole is exposed to direct flux. As the comet retreats from equinox, the flux received by the ever exposed south-pole depends on the tilt angle, making the small tilt angle pole receive only a fraction of the flux received by a high tilt pole. The lowest value of the maximal surface temperature is less than $130^{\circ} \mathrm{K}$. At such temperatures crystallization of amorphous water ice proceeds very slowly: the characteristic timescale is of the order of days, competing with the dynamical (orbital) timescale. It is thus possible that amorphous ice be retained at (or very nearly below) the surface of a very confined area, provided that the tilt angle remains unchanged. On the other hand, this temperature 

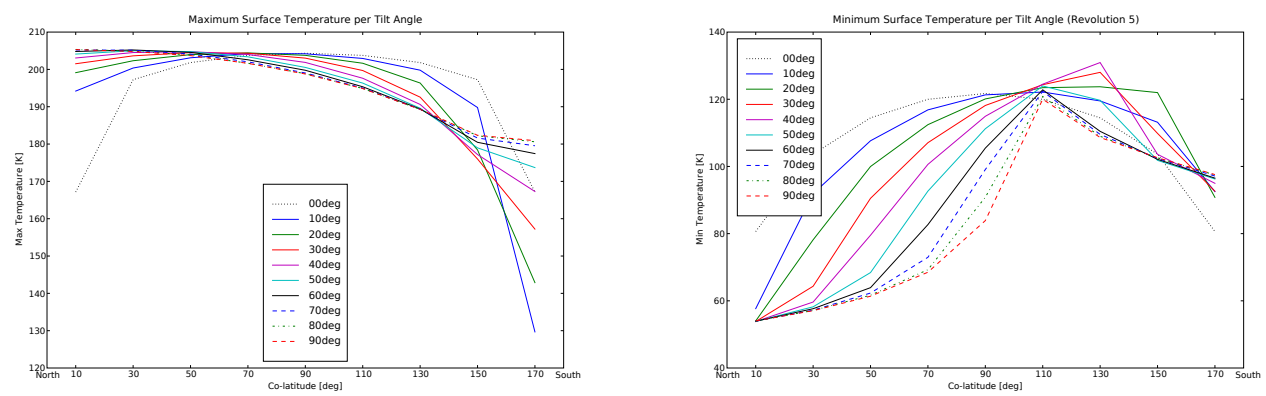

Fig. 9. Maximum and minimum local surface temperatures for 10 different spin-axis tilts. Extremum temperatures vary only with co-latitude, and are greatly affected by tilt angle. The lowest value of the maximum temperature is obtained at the southern pole for small tilt angle. The lowest temperatures (over the $5^{\text {th }}$ revolution) are found at the north pole, where the highest maximum temperature occurs.

is far too high for ices of volatile substances (certainly $\mathrm{CO}, \mathrm{CH}_{4}, \mathrm{~N}_{2}$, but also $\mathrm{CO}_{2}, \mathrm{NH}_{3}, \mathrm{HCN}$ ) to survive insolation at the surface. These molecules observed to be ejected by comet nuclei - must, therefore originate in deeper layers or be released by crystallization of gas-laden amorphous water ice. It is, therefore, inconceivable that pristine material be found on the surface of comets of the Jupiter family type.

Minimum temperatures (right panel of Fig. 9) are lowest for the north pole, which is allowed to cool for most of the comet's orbit. Temperatures drop below $60^{\circ} \mathrm{K}$, which may render the surface and the outer layers almost completely inert.

To further study the influence of small tilt angles on sub-surface crystallization, a high-resolution model of comet $67 \mathrm{P} / \mathrm{C}-\mathrm{G}$ was computed, adopting a $15^{\circ}$ tilt angle. This high-resolution run of the model took 3 months of calculations on a $3.0 \mathrm{Ghz} 64$ bit Pentium-4 based PC. Therefore, it was not conducted exhaustively for each tilt angle. The model started with 50\% amorphous ice that was allowed to crystallize. Fig. 10 shows the crystallization cross-section pattern (top) and the temperatures for the same area (middle) at 1.5 AU postperihelion. It is shown that the south-pole crystallization front did not exceed depth of $3 \mathrm{~m}$ (during the entire run of the model the front did not advance beyond $4 \mathrm{~m}$ ), in contrast to a model with $40^{\circ}$ tilt angle (not shown here) that reached a depth of $10 \mathrm{~m}$ within the same period. The north-pole crystallization front did not exceed the depth of $2 \mathrm{~m}$ in both models, although maximum temperatures were the highest. This happened due to the rapid exposure of the pole to the solar flux. When the pole is hidden from the sun (most of the time), the cooling boundary conditions with only small amount of heat that has been absorbed during solar exposure, prevent the deeper temperature from increasing, and thus crystallization propagates only to a shallow depth. This may indicate that pristine material may be found at the north-pole at small depths but not at the surface. 

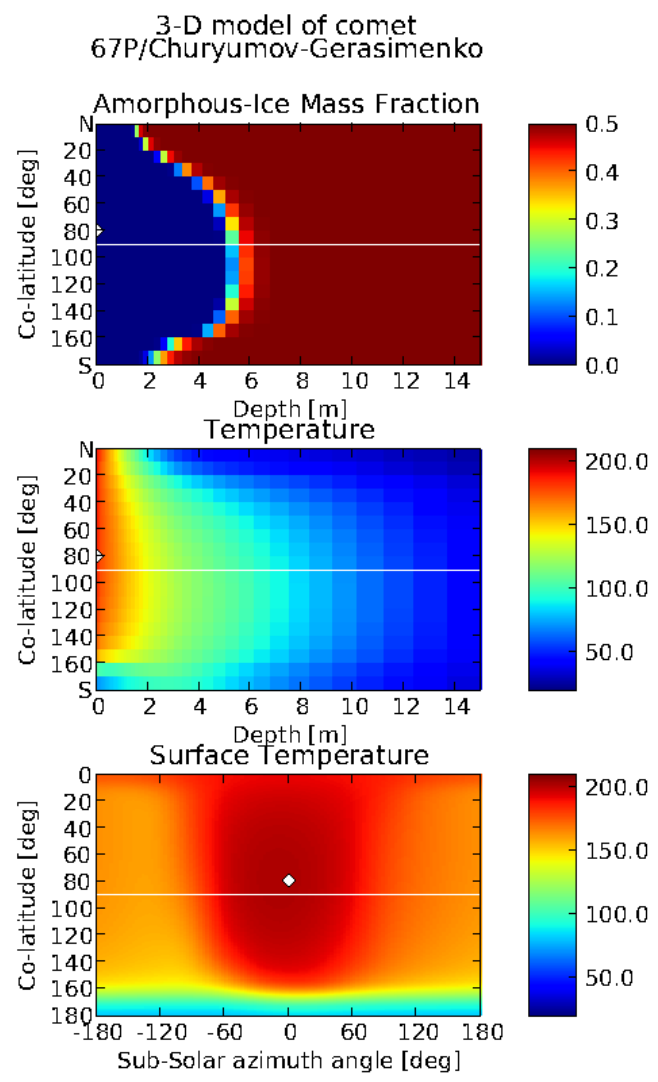

Fig. 10. Surface temperature and advance of the crystallization front for comet $67 \mathrm{P} / \mathrm{C}-\mathrm{G}$ with $15^{\circ}$ tilt angle at $1.5 \mathrm{AU}$ post-perihelion. The model was started with $50 \%$ dust and $50 \%$ amorphous ice with $H_{z}=0.01$ and the amorphous ice was allowed to crystallize. Top panels show the amorphous ice mass fraction and temperature as function of co-latitude angle and depth, with arbitrary azimuth. The crossing white line represents the equatorial plane and the white dot at the ordinate represents the sub-solar co-latitude angle. Surface temperature (bottom) is shown with same equatorial and subsolar markings.

We note that our present model does not account for possible recession of the surface due to sublimation of the ice. This effect would distort the sphericity of the model (numerical grid). On the other hand, as stated above, most of the surface of comet nuclei is covered by a dust mantle, presumably of high porosity, and water vapor is produced and ejected from an ice-rich layer lying beneath the dust mantle. Therefore, the process of erosion may be more complicated than just simple ablation and requires separate investigation. The point we wish to stress in the present calculation is that amorphous ice may lie very close to the nucleus surface under appropriate conditions, although it would not be detectable on the surface itself. 


\section{Summary and Conclusions}

We have developed a fully 3-D thermal evolution code for comet nuclei that may, in fact, be applied to any small body of the solar system, small enough for self-gravity to be negligible. In the present paper, which is the first of a series, we have tested the code with respect to analytical solutions, as well as 1-D and quasi-3-D comet nucleus models, and found excellent agreement.

We computed models of comet $67 \mathrm{P} / \mathrm{C}-\mathrm{G}$, adopting a $1: 1$ mass ratio of ice to dust. In this case, surface temperatures are mainly determined by the ice, through the strongly temperature-dependent sublimation term of the boundary condition. Assuming a spin axis tilt of $40^{\circ}$, we obtained a maximum surface temperature of $205^{\circ} \mathrm{K}$ and a minimum temperature of $54^{\circ} \mathrm{K}$.

We have investigated the effect of the spin axis tilt on the surface temperature distribution and found that conditions for preservation of pristine amorphous ice and moderately volatile species at shallow depths is possible for low tilt angles, when some surface areas are shielded from insolation. This is despite lateral heat conduction driven by the strong temperature variations at the nucleus surface. Although not shown here, more distant comets with low tilt angles could retain unprocessed surface amorphous ice.

A fully 3-D comet nucleus model opens a wide range of possible subjects of study, involving inhomogeneities, both innate and evolutionary, which appear to be so common to comets. We shall address some of them in upcoming papers.

Acknowledgments Support for this work was provided by the Israel Science Foundation grant No. 942/04.

\section{References}

A'Hearn, M. F., Belton, M. J. S., Delamere, W. A., Kissel, J., Klaasen, K. P., McFadden, L. A., Meech, K. J., Melosh, H. J., Schultz, P. H., Sunshine, J. M., Thomas, P. C., Veverka, J., Yeomans, D. K., Baca, M. W., Busko, I., Crockett, C. J., Collins, S. M., Desnoyer, M., Eberhardy, C. A., Ernst, C. M., Farnham, T. L., Feaga, L., Groussin, O., Hampton, D., Ipatov, S. I., Li, J.Y., Lindler, D., Lisse, C. M., Mastrodemos, N., Owen, W. M., Richardson, J. E., Wellnitz, D. D., White, R. L., Oct. 2005. Deep Impact: Excavating Comet Tempel 1. Science 310, 258-264.

Benkhoff, J., Jun. 1999. Energy balance and the gas flux from the surface of comet 46P/Wirtanen. Planetary and Space Science 47, 735-744. 
Benkhoff, J., Boice, D. C., Jul. 1996. Modeling the thermal properties and the gas flux from a porous, ice-dust body in the orbit of $\mathrm{P} /$ Wirtanen. Planetary and Space Science 44, 665-673.

Biver, N., Bockelée-Morvan, D., Colom, P., Crovisier, J., Germain, B., Lellouch, E., Davies, J. K., Dent, W. R. F., Moreno, R., Paubert, G., Wink, J., Despois, D., Lis, D. C., Mehringer, D., Benford, D., Gardner, M., Phillips, T. G., Gunnarsson, M., Rickman, H., Winnberg, A., Bergman, P., Johansson, L. E. B., Rauer, H., 1999. Long-term Evolution of the Outgassing of Comet Hale-Bopp From Radio Observations. Earth Moon and Planets 78, $5-11$.

Carslaw, H., Jaeger, J., 1959. Conduction of Heat in Solids, 2nd Edition. Oxford University Press.

Chesley, S. R., Nov. 2004. An Estimate of the Spin Axis of Comet 67P/Churyumov-Gerasimenko. Bulletin of the American Astronomical Society $36,1118-+$.

Cohen, M., Prialnik, D., Podolak, M., Mar. 2003. A quasi-3D model for the evolution of shape and temperature distribution of comet nuclei-application to Comet 46P/Wirtanen. New Astronomy 8, 179-189.

Cowan, J. J., Ahearn, M. F., Oct. 1979. Vaporization of comet nuclei - Light curves and life times. Moon and Planets 21, 155-171.

Enzian, A., Cabot, H., Klinger, J., Mar. 1997. A 2 1/2 D thermodynamic model of cometary nuclei. I. Application to the activity of comet 29P/Schwassmann-Wachmann 1. Astronomy and Astrophysics 319, 9951006.

Enzian, A., Klinger, J., Schwehm, G., Weissman, P. R., Mar. 1999. Temperature and Gas Production Distributions on the Surface of a Spherical Model Comet Nucleus in the Orbit of 46P/Wirtanen. Icarus 138, 74-84.

Fanale, F. P., Salvail, J. R., Dec. 1984. An idealized short-period comet model - Surface insolation, H2O flux, dust flux, and mantle evolution. Icarus 60, 476-511.

Ghormley, J. A., 1968. Enthalpy change and heat-capacity changes in the transformations from high-surface-area amorphous ice to stable hexagonal ice. Journal of Chemical Physics 48, 503-508.

Gutiérrez, P. J., Ortiz, J. L., Rodrigo, R., López-Moreno, J. J., Mar. 2000. A study of water production and temperatures of rotating irregularly shaped cometary nuclei. Astronomy and Astrophysics 355, 809-817.

Herman, G., Podolak, M., Feb. 1985. Numerical simulation of comet nuclei. I - Water-ice comets. Icarus 61, 252-277.

Julian, W. H., Samarasinha, N. H., Belton, M. J. S., Mar. 2000. Thermal Structure of Cometary Active Regions: Comet 1P/Halley. Icarus 144, 160171.

Keller, H. U., Arpigny, C., Barbieri, C., Bonnet, R. M., Cazes, S., Coradini, M., Cosmovici, C. B., Delamere, W. A., Huebner, W. F., Hughes, D. W., Jamar, C., Malaise, D., Reitsema, H. J., Schmidt, H. U., Schmidt, W. K. H., Seige, P., Whipple, F. L., Wilhelm, K., May 1986. First Halley multicolour 
camera imaging results from Giotto. Nature 321, 320-326.

Lamy, P. L., Toth, I., Fernandez, Y. R., Weaver, H. A., 2004. The sizes, shapes, albedos, and colors of cometary nuclei. pp. 223-264.

Prialnik, D., Bar-Nun, A., Feb. 1987. On the evolution and activity of cometary nuclei. Astrophysical Journal 313, 893-905.

Prialnik, D., Benkhoff, J., Podolak, M., 2004. Modeling the structure and activity of comet nuclei. Comets II, pp. 359-387.

Schleicher, D. G., Millis, R. L., May 2003. Results from Narrowband Photometry of ROSETTA's New Target Comet 67P/Churyumov-Gerasimenko. Bulletin of the American Astronomical Society 35, 970-+.

Schmitt, B., Espinasse, S., Grim, R. J. A., Greenberg, J. M., Klinger, J., Dec. 1989. Laboratory studies of cometary ice analogues. Tech. rep.

Sunshine, J. M., A’Hearn, M. F., Groussin, O., Li, J.-Y., Belton, M. J. S., Delamere, W. A., Kissel, J., Klaasen, K. P., McFadden, L. A., Meech, K. J., Melosh, H. J., Schultz, P. H., Thomas, P. C., Veverka, J., Yeomans, D. K., Busko, I. C., Desnoyer, M., Farnham, T. L., Feaga, L. M., Hampton, D. L., Lindler, D. J., Lisse, C. M., Wellnitz, D. D., Mar. 2006. Exposed Water Ice Deposits on the Surface of Comet 9P/Tempel 1. Science 311, 1453-1455. 